\title{
NORDUNET: THE ROOTS OF NORDIC NETWORKING
}

\author{
Rolf Nordhagen \\ USIT, Centre for Information Technology Services, University of Oslo, Norway; \\ rolf.nordhagen@usit.uio.no
}

Abstract: NORDUNET began as an informal cooperation between Nordic "networkers" in 1980. With support from the Nordic Council of Ministers, a NORDUNET project for a common Nordic academic network began in 1985. Mats Brunell (Sweden) and Einar Løvdal (Norway) led the work. Originally based on existing interim services of EARN, DECnet and ISO OSI support, lack of services led to complete reorientation in 1987. With bridges running Ethernet over slow lines, a Nordic-wide Ethernet connecting major nodes in the countries linked national Ethernets to a common node at KTH, Stockholm. The major services of the time, X.25, EARN and RSCS, DECnet, and TCP/IP, were connected in through switches, bridges and routers called "the NORDUNET plug". The operational network NORDUnet, a first international multi-protocol network, began services in 1988 and officially opened in 1989. Major links to the US NSFnet and European networks connected to the KTH node. The project had a strong impact on Nordic networking competence that influenced the European move to TCP/IP services in opposition to the prevailing adherence (politically supported) to ISO OSI. Over time, TCP/IP won the "protocol war". The early introduction of TCP/IP gave the Nordic area a head start in internet penetration, still reflected in the countries being in the front of public use of the internet. A major lesson was the success of Nordic cooperation on all levels, through sharing of responsibilities, joint development of competence and creation of enthusiasm. NORDUnet is today owned by the national ministries, run through cooperation by the national networks, and able to supply the Nordic academic internet with exceptionally cost-effective bandwidth to all major international networks such as Startap and Geant.

Key words: Computer communication, Nordic cooperation, internet penetration, multiprotocol networks 


\section{INTRODUCTION}

The history of NORDUNET, the project, is one of cooperation and friendship. It is also the history of the world's first international, multiprotocol network and on how the NORDUNET team won the "great European protocol war". This version of the story reflects a personal background from the University of Oslo and its approach to computer networking.

\subsection{The beginning}

The arrival of network projects on the computer scene in the late $60 \mathrm{~s}$ and beginning 70 s fueled our beginning interest. The emergence of powerful standards to construct networks, based on the OSI-model, inspired our technical people. The phrase "Open networks" had the meaning of "An open network is a network following an open, common standard". In addition, after years of dependence on the large mainframe, we escaped from the rigors of batch processing by the affordable minicomputer.

In Oslo, we did remote stations to mainframes, and transferred to timesharing in the mid-70s, spurring interest on terminal networks. Also, after initial study groups, Norwegian universities and research institutes initiated a national academic network project, UNINETT, which by 1978 had established a packet switched service and by 1979 had access to all major university machines, all solidly based on the X.25 protocol. Similar efforts got under way in Sweden where SUNET, established in 1980, created a service also based on connecting the institutions local terminal networks to the public X.25 network. As the service gave international dial up connectivity, we could reach out internationally, also to US and Canadian networks.

\subsection{The first ARPANET connection}

In 1973, as the first outside the U.S., ARPANET was connected to a seismic array project NORSAR at Kjeller, Norway, by a $2.4 \mathrm{~kb} / \mathrm{s}$ satellite link. In the Cold War, it was clear that the Norwegian bedrock was a perfect listening station for nuclear arms testing. A terrestrial line soon extended the connection itself to the University College of London.

The later history of how this connection evolved into a full internet service for a few informatics institutes in Norwegian universities is in itself fascinating. In reality, the availability of the TCP/IP suite of protocols under Berkeley UNIX moved the connectivity out of the Kjeller labs. In retrospect, sadly, the activities never became part of the mainstream efforts 
leading to a national network, mainly due to close dependence on a few enthusiasts with little support from their own institutions. At that time, all official network projects had their foundations on the OSI protocols. Thus, the enthusiasts did not accept them as suitable partners; rather, they viewed them as competitors.

However, by 1983/84 a number of institutes in Norway enjoyed full internet access, but not available nationally or accessible from other countries, in contrast to the by then established but limited X.25 services. This became a general envy and a strong pressure on major projects to supply similar services.

It is an interesting twist of history that when the satellite connection, then part of SATNET, was finally removed in 1987 and the institutes lost their internet connectivity, we were only a few months away from bringing up the identical NORDUnet service properly organized in a common network.

\subsection{The early introduction of computer communication}

In my opinion, another major inspiration for Nordic networking was the early emergence a powerful communication service, the Stockholm KOM program. The university computer centre in Stockholm, (they called themselves QZ as these letters were used by no one else) installed a timesharing DIGITAL DEC-10 in the early 70s. Jakob Palme, working with the DEC-10, became inspired by the communication ideas of Murray Turoff at the New Jersey Institute of Technology ("The network nation") and wrote what he called a computer conferencing system he named KOM. Today we would class it as a breed between a mail system and a "news"-system, with features that even today were superlative to presently used systems. It was an instant success and over the years, it was reachable by remote login via the growing international X.25 services. It finally became a European meeting place.

In Oslo, we installed a DEC-10 in 1976, running the first timesharing service in Norway. Close cooperation sprung up between the Oslo and Stockholm installations, based on good personal relations between the Swedes and Norwegians involved. In 1978, we were able to install KOM in Oslo, again an instant success, which really brought home to us the qualities of computer communication. Even if was a centralized system, as the remote login services improved in UNINETT, it became an important national service. 


\section{INTRODUCING NORDIC COOPERATION}

Then enter true Nordic cooperation. To strengthen the bonds between the Nordic countries in the wake of the WW2 disruptions, several councils emerged and as examples, they introduced a Passport Union and a common labor market. A Nordic Council of Ministers, being the responsibility of the Nordic governments, had created an office for coordination in research called Nordforsk. The director, Bjørn Grønlund, caught onto the idea of Nordic cooperation in computer networking. In 1980, he convened a workshop in Tellberg, Dalarne, Sweden, where all major network projects in the Nordic countries were invited. To me, this was an exhilarating experience, to meet many fellow enthusiasts. The various network adherents banded together, dreaming of Nordic-wide DECnets, IBM-nets, and common X.25 access. This workshop continued as meetings under the name of NORDUNET, Nordic University Networks, circulating among the nations, Denmark, Norway, Finland, and back in Göthenburg, Sweden in 1984.

\subsection{The EARN network}

In 1984, another important part of the play began. The computer firm IBM found that they had a thriving computer network community based on BITNET running among large IBM installations initially on the East coast of the U.S. They saw an opportunity to get a foothold among the less organized European networks, and donated a network, EARN, European Academic Network, based on BITNET and running on international leased lines sponsored by IBM. This offer was very well received and with some of the larger Nordic IBM installations, became an important service.

\subsection{And then: The NORDUNET program}

By 1983, the NORDUNET meetings, apart from inspiration, had produced no concrete actions. Nevertheless, the time was ripe to go one-step further, actually to apply for Nordic money for network activities. The major network groups agreed to launch a true NORDUNET network project, and a group, led by Arild Jansen, University of Oslo and Jorgen Richter, NEUCC, Denmark received the task to edit together a full project application. The meeting in 1984 approved the application "NORDUNET a Nordic program of action in data-communication" and later in 1984, NORDFORSK presented the application to the Council of Ministers.

As it turned out, the ground was well prepared. The Council of Ministers found they had extra money due to favorable exchange rates, and wanted 
ideas on inter-Nordic actions in computing. At one stage, Bjørn Grønlund suggested a supercomputer centre. "How much will that cost," they asked. Bjørn answered, 100 million kroner. Next question: "Can we do anything less expensive?" Yes, we can create a common Nordic computer network at a cost of 10 million kroner. Thus, in May 1985, the Nordic Ministers of Culture and Education granted 9.2 million NOK to a Nordic University Network, NORDUNET, and directed NORDFORSK to act as secretariat for the program. Hence, NORDUNET, the network project was born.

\subsection{The initial requirements}

1. The program will run for four years and establish a stable, operating computer network, connecting the Nordic Educational and Research institutions (NR\&Es) and offering easily accessible communications services.

2. The program shall open for electronic exchange of letters, documents, and data and make a common use of data-resources, programs, and databases available throughout the Nordic area. Users shall be given access to Nordic and international networks and information services.

3. NORDUNET should not establish its own network, but build on existing university networks. Use should be made of the Nordic telecommunication networks, and the network be based on international standards for tele- and data-communication.

4. This will establish a common Nordic infrastructure for the NR\&E community by connecting the national nets, and new competence and services will be created for the smaller communities. The same opportunities for collaboration and information exchange as in other European countries and in the North American research communities will be made available.

5. The resources to achieve these goals will in the main have to be found in the national network organizations, with the local, university service providers, charged with the day-to-day .operation of the net.

6. Other important, positive arguments for the decision were the clear cooperative nature of the program and the belief that a computer network would greatly increase bridging the large geographical distances characteristic in the Nordic countries. Thus, opportunities for research in 
remote communities would be greatly improved. All arguments we have used since the beginning of networking, and still use.

\subsection{The NORDUNET work begins}

After the administrative dust settled, a steering group was set up and the work began, in earnest in 1986. Initially Bjørn Grønlund, NORDFORSK, administered the project; later it was transferred to Stocholm QZ and Mats Brunell. A technical coordinator/manager was hired from the University of Oslo, Einar Løvdal. (See Figure 1.) It is largely due to the efforts of these two energetic persons that NORDUNET achieved its results. Actually, two friendly organizations picked their two best people and set them to the task.

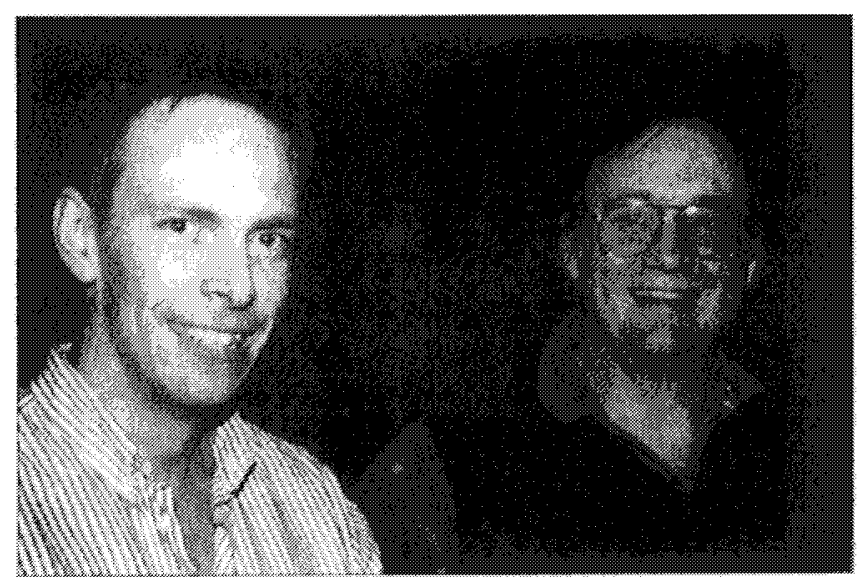

Figure 1. Mats Brunell and Einar Løvdal

At the time, people believed that any common service had to be based on the connection oriented OSI protocols. This even became a hot political issue, as the European commission strongly backed OSI as a platform for European networking. As is known in retrospect, this unfortunate involvement killed a European networking hardware industry, as the policy did not survive. Also at the time, a number of European initiatives emerged, RARE, a cooperation similar to NORDUNET, but aimed at unifying European networking, an EARN cooperative body, and COSINE, an effort to coordinate the development of OSI-based services. RARE/COSINE initiated a common network project IXI. Because of the NORDUNET project, we already had a clear benefit by financially being able to join these bodies as joint Nordic members, something most of the countries would have difficulties in doing separately. 
Initially the task of creating a common network service seemed chaotic. At that time, a number of networks were in operation, local and private, such as the national network groups ran private X.25 networks, accessed by Telecompany PAD-services. All major institutions also ran Ethernets as isolated, local networks. Coordinate common services seemed a formidable task.

As a start, popular services were selected and made available Nordicwide. As they had to operate as separate services, the efforts were strategically named "Interim" services, on the way to a common service.

The EARN services joined and accessed the European wide services through UNI-C in Denmark. A joint DECnet effort worked to connect the high-energy physics institutions to HEPNET, originating in CERN. For the X. 25 based services, an X.400 based message-handling system, EAN, from University of British Columbia was already part of UNINETT; it became available in the other countries through licensing agreements. The UNINETT group did the coordination. This effort led to the group also got responsibilities for a similar service in Europe.

\subsection{The protocol challenges and reorientation}

An overall effort to develop further services became a major part of the program. Work began with partners in several major network groups in the countries. The big question of the time was what suite to base the services on. The political pressure as mentioned, as well as the belief of the European technical expertise, was to use the CCIT recommended OSI style connection oriented protocols such as X.25. However, services were slow in development. Additionally, stable standards were not yet available; the work in the ISO standardization in CCIT progressed slowly and continuously as it was close to finalization. This led to a reorientation of original NORDUNET goals. The effort to develop services independently was regarded as unrealistic. The interim solutions with services based on the separate, not yet connected, private network would continue to be supported, but prepared for OSI-migration. Furthermore, services were to be as much as possible based on industry-supported products. Interim solutions to connect to international networks, such as EARN, UUCP, DECnet and INTERNET should be sought. Moreover, to prepare for OSI-migration, pilot services emerged and organized on a Nordic level. NORDUNET should actively work in the European OSI-efforts in RARE and COSINE, to strengthen Nordic competence and coordinate Nordic and European efforts.

Several subprojects began. One was an effort to develop a file-transfer pilot service based on the FTAM protocols. This turned out to be highly resource consuming. One discussion was on choosing the JANET colored 
book services; other efforts were to use the ISODE suite of programs to run X.25 over IP. Progress was still enthusiastic, but slow. Furthermore, the X.25 pilots showed the service to be far less stable than expected; they were slow and resource consuming. The future for a full, reliable set of standards seemed still to be years off, JANET talked about still ten years, which would mean the NORDUNET project would be in only a pilot phase for the duration of the four-year period.

Since the technical challenges were still formidable, the setbacks were regarded as just that, challenges. The goal of a common network service was still a great source of inspiration. Since they kept project costs at a minimum, the main expenses being part time management and modest coordination support, mostly travel, licensing, and financing the effort was not a severe problem. Therefore, the work went doggedly on.

\section{THE NEW DIRECTION AND BREAKTHROUGH}

However, in 1987 a number of major technical and organizational happenings came together to change the networking world for NORDUNET. Firstly, IBM announced that the support of the (expensive) leased lines for the Nordic EARN-network had to be cut, thus putting heavy pressure on the NORDUNET resources for continuation. Secondly a major technical breakthrough occurred, bridges transmitting Ethernet over slow, leased lines became available, the Vitalink bridges. These became an immediate success. The University of Oslo quickly managed to get these bridges for connecting the Ethernet on the main campus with local networks in their institutions spread all over the Oslo area. Prompted by the need to take over a Nordic wide leased line network, at the time linking major nodes in four countries, they quickly understood that such a network suitably supplied with Vitalink bridges in each node, could constitute a Nordic wide Ethernet. Moreover, since just as the local Ethernets supported the protocols that the various private networks required, the Nordic Ethernet, suitable equipped with routers, gateways and bridges also could support the same networks. The meetings in which this understanding first became clear, was I firmly believe, personally my high point in our NORDUNET work. The gate to realizing our original goal had opened.

Things happened quickly. The new project was aptly named X.EARN, a strategic name to underline that we just had an improved and continued effort to replace the EARN network, hiding the perhaps controversial fact that we aimed at supporting other network protocols and even straying away from the orthodox OSI line. A brain trust of prominent networkers from four countries wrote a X.EARN project plan, adopted early in 1988 by the 
NORDUNET steering group, and subsequently by the national networks. The report covered a number of important points, as it turned out, all solvable by common efforts.

\subsection{The NORDUnet becomes operational}

The techniques, of course, are well known today. An Ethernet based on bridges connected national nodes in each country, itself a bridge between the common net and a national Ethernet. Thus, the inter-Nordic nets stayed clean from national connections. On the national Ethernet, again local Ethernets connected the member institutions. Anywhere on the Ethernets, but in practice nationally or institutionally, they could connect routers, bridges, and gateways to the various supported protocols and services.

The concept was formulated by Einar Løvdal with his NORDUNET "plug" (Figure 2) that carried the basic X.25 service, EARN and RSCS, DECnet, and TCP/IP.

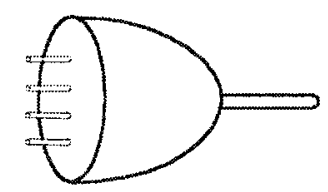

Figure 2. Løvdal's "plug" concept

Note that the challenge was not directly technical; running these services over Ethernets was well in use. The challenge was organizing the strategic placing of the various connecting devices, and coordinating Nordic-wide operation. Management of the various services and the localization of major gateways were awarded by contract to competent groups in the countries. The principle of a distributed service organization was introduced.

Another helpful event coincided with establishing a private Nordic net. The Nordic telecom companies created a joint company providing one-stop shopping for inter-Nordic lines called Scantele. The contracting for the lines between the major Nordic sites (Copenhagen, Helsinki, Stockholm, and Trondheim) became reasonably easy. The network ran on $64 \mathrm{~kb} / \mathrm{s}$; Iceland was on a separate connection running originally at $9.2 \mathrm{~kb} / \mathrm{s}$. During 1988 , the network was established, with the necessary equipment in place, and named NORDUnet (with the lower-case letters) indicating the operational net. The major node on the Nordic Ethernet was located at KTH (Royal Technical Institute) in Stockholm. 


\subsection{Worldwide connectivity}

An important strategy was to create worldwide connectivity for Nordic research through international nets. Excellent contacts with US universities and research groups made it possible to connect via a $56 \mathrm{~kb} / \mathrm{s}$ satellite line to the supercomputer centre in Princeton, New Jersey, and then onto NSFnet, thus connecting the Nordic academic area into the budding internet and the TCP/IP based services. Furthermore, important European networks were connected in Stockholom, EARN, HEPnet, CERN, and EUnet. The operations centre in $\mathrm{KTH}$, Stockholm was the common connection point, were the concept of peering was introduced. Thus, NORDUnet and EUnet provided backup for their transatlantic connections. Over the years, the KTH group developed the node into an important European interchange, GIX, connecting other countries such as those in the Baltics and Poland.

Thus, the world's first, truly international and multi-protocol network was created. Although services already had been available, the official opening was in October 1989, at a NORDUNET meeting outside Stockholm. Figure 3 illustrates its expansion.

\subsection{When TCP/IP took over}

The NORDUNET program itself went on, working hard on harmonization of mail and other services. In addition, the strategy of OSImigration was still followed, with participation in the European IXI effort to create an OSI-based net. Projects on running connectionless over OSI were launched. Remember, that at the time, US standardization bodies were also true believers in OSI. With extensions, the program lasted until 1991.

All this is now history. NORDUnet, the network was arranged to be self sustained, originally run by the national network, today a limited company owned by educational institutions in the countries. It is still based on a small secretariat, with the actual operational work being contracted to the national networks. By joint purchase of bandwidth, the Nordic academic networks can negotiate for very cost effective agreements. The area has some of the heftiest transatlantic connections in Europe. 


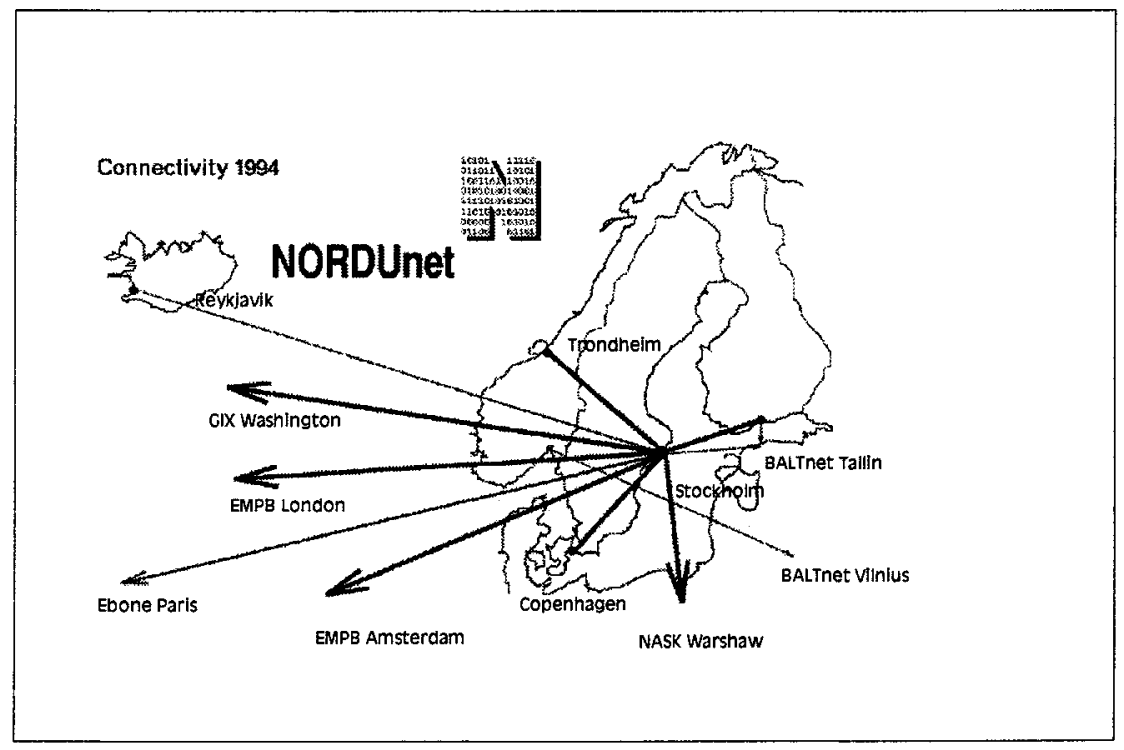

Figure 3. NORDUNET in October 1989

From opening up the network to multiple protocols, a natural migration took place. It became apparent early that the TCP/IP based services were far superior to (almost non-existing) services on other nets. Important cooperation in Europe, working with US and Canadian collaborations, such as the high-energy community, were urging for transition to TCP/IP. Einar Løvdal, while presenting his plug at the RARE meeting in Trieste in 1989, made a plea for European wide introduction of TCP/IP. He received very mixed reactions. The conviction that Europe should forge ahead with OSIbased services was solidly rooted in the then existing largest national networks, JANET in the UK and DFN in Germany.

Again, we know the history. One by one, the other services disappeared. The last to go was X.25, taken down in NORDUnet in 2001. By giving all protocols equal opportunity, we won the protocol war as the best services survived-TCP/IP and the internet.

\subsection{Not only a network}

NORDUNET created more than a network. The extensive use of interNordic workgroups in network management and protocol development created competence on many levels. This had a catalytic effect on national networks and introduction of services. Teaching network technologies became part of university curricula. All this, further led to an early 
commercial introduction, first by startups, later by the National Telecom companies.

Joint activities created international recognition and status in international bodies. The first DNS-rootserver outside the US was in Stockholm. Members of the Stockholm team, Mats Brunell and Bernhard Stockman, were central in establishing RIPE as a European internet coordinator. They participated in forming the work of IEPG, and in IETF Operations WG. A number of NORDUNET experts were active in IETF working on MIME. Working in RARE WG8-management, the NORDUNET team received support for an open policy on protocols and services, similar to the NORDUnet policy, opening up for the introduction of $\mathrm{TCP} / \mathrm{IP}$ while at the same time working on ISO OSI. A policy at the time regarded as highly controversial but important to move Europe on to the internet. Figure 4 shows the phenomenal exponential growth of NORDUnet network links in the US.

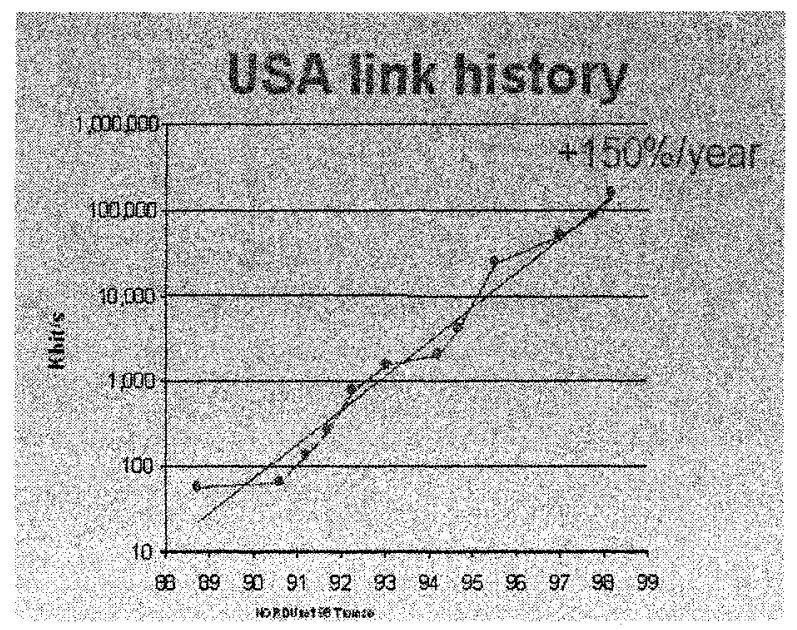

Figure 4. NORDUnet growth in the US

\subsection{The roots}

Thanks to the early access to the internet, the growth of network nodes in the Nordic area has been spectacular. In October 1991, 14,000 of the 32,000 IP-nodes in Europe were located in the Nordic area. Several major European networks were years behind in providing IP-based services. Even after commercial services has vastly surpassed NORDUnets early introduction of internet access, the head start is still reflected in the statistics, with the Nordic countries for years have been among the ten on top as to internet penetration in their populations, in 1999 actually among the seven 
with Canada and the US. Truly, NORDUNET and NORDUnet are the roots of Nordic networking.

Table 1. Nordic internet penetration $\%$ per capita

\begin{tabular}{lccc|cccc}
\hline & 1999 & & 97 & \multicolumn{4}{c}{1999} \\
\hline 1 & Canada & 42,82 & 7 & 8 & Australia & 34,33 & 5 \\
2 & Sweden & 41,42 & 8 & 9 & Singapore & 31,08 & 9 \\
3 & Finland & 40,80 & 1 & 10 & N.Zealand & 26,49 & 6 \\
4 & U.S. & 40,65 & 4 & 11 & Netherland & 25,56 & 13 \\
5 & Iceland & 40,35 & 3 & 12 & Switzerland & 24,58 & 11 \\
6 & Denmark & 39,60 & 10 & 13 & United Kingdom & 23,64 & 12 \\
7 & Norway & 37,96 & 2 & & & & \\
\hline
\end{tabular}

\section{CONCLUSIONS}

As a conclusion, here follows a number of statements, comprising what we call the NORDUNET lesson. The challenge became "communication is cooperation". The highlights are:

- Services could not be done by one provider alone

- The necessary level of competence could not be reached on a country by country basis

- Institutional groups too small both in people with interest and knowledge resources and demanding users

- Development cooperation required on all levels

- Cooperation gives weight to international presence

The NORDUNET lesson is as follows.

- Many institutions scattered across several countries worked together by each getting major responsibilities

- Distributed projects created joint enthusiasm and work towards common goals

- Shared responsibilities

- All received benefit from building competence

- Network communication is working together 
Hence, we can use the following syllogism.

\section{Networks are communication \\ Communication is Cooperation \\ Networks are Cooperation}

\section{FINAL REMARK}

Moreover, the attitude to know carefully what you aim to do, and to take responsibility for the consequences, is brought out by the NORDUNET slogan:

$$
\begin{gathered}
\text { "Skalat madr rúnar rísta, nema ráda vel kunni" } \\
- \text { Egill Skallagrimsson }
\end{gathered}
$$

Written by the greatest Icelandic poet of the 11th century, a simple translation says, "If man draws runes, he must know what he is doing". However, they tell me the meaning is more subtle in that he must realize the power and meaning of his runes. As we in networking are reminded when we realize what we have unleashed on the world, the internet, the web, and a deluge of content.
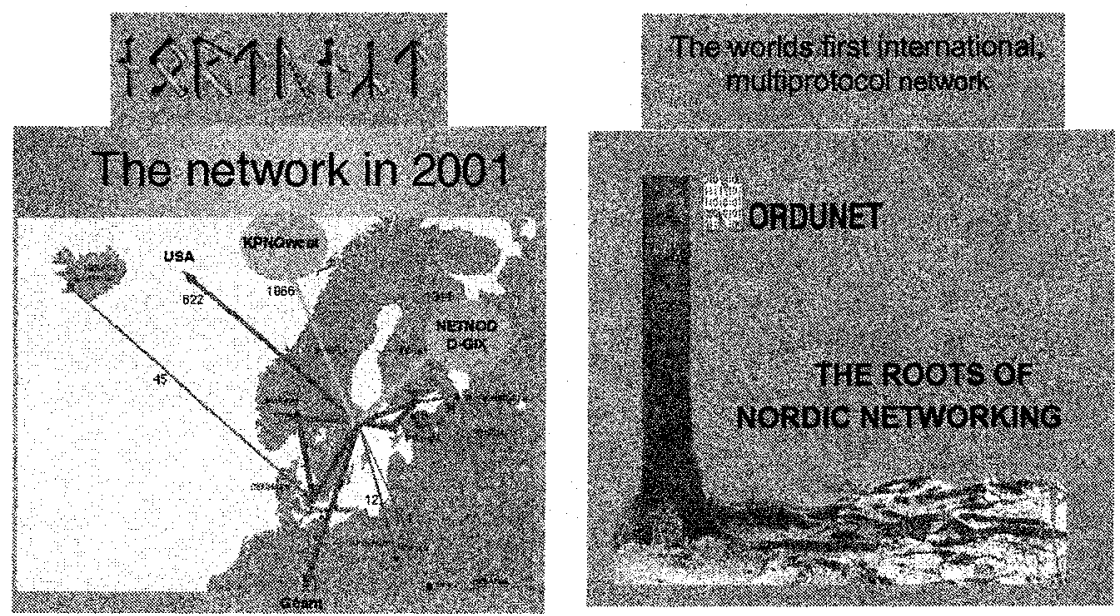